\title{
Organization Conditions Enabling Employee Empowerment and the Moderating Role of Individual Personalities
}

\author{
Sharon Pande ${ }^{1} \&$ Udayan Dhar $^{2}$ \\ ${ }^{1}$ Human Resources and Behavioral Sciences at the School of Business Management, NMIMS University, India \\ ${ }^{2}$ School of Business Management, NMIMS University, India \\ Correspondence: Sharon Pande, Human Resources and Behavioral Sciences at the School of Business \\ Management, NMIMS University, India. E-mail: sharon.pande@nmims.edu
}

Received: July 21, 2014

Accepted: August 22, 2014

Online Published: September 25, 2014

doi:10.5539/ijbm.v9n10p70

URL: http://dx.doi.org/10.5539/ijbm.v9n10p70

\begin{abstract}
Empowerment is considered a key driver of organization growth. Companies are realizing that in today's knowledge economy, high levels of supervision and direction actually hamper the full utilization of the employees' potential to contribute towards the overall organization's objectives.

Data was gathered from 243 employees to understand what really counts as empowerment and what impact it has on their effectiveness at work, their levels of innovation, leadership skills, commitment to the organization and their ability to manage stressful situations at the workplace.

The research shows that except stress management, a highly empowered workplace has a strong positive correlation to all the above mentioned factors- at least as far as employee perceptions are concerned. The more interesting fact emanates when one tries to see if the personality of the employees also has any impact in all of this.

The results clearly show that employees with higher levels of core self evaluation, self-efficacy, risk taking abilities, pro-activeness and an internal locus of control will do best only when provided with a work culture that allows for their empowerment. In fact, wider the gap between the employees personality type and the work environment in terms of empowerment, the poorer will be the outcome on all these parameters-effectiveness, innovation, leadership skills, commitment and stress management. Interestingly, the reverse isn't true. Providing a more empowered environment than what employees are thought to be able to handle (in case of employees with lower levels of self efficacy, etc.) does not lead to any similar negative consequences.
\end{abstract}

Keywords: employee empowerment, individual personalities, personality and empowerment, organization conditions and organizational outcomes

\section{Introduction}

Feist and Feist, (2008), have defined personality as a pattern of moderately enduring traits and exclusive characteristics that give not only consistency to a person's behavior, but, also individuality. Several models and components of personality have been proposed. The ones that are considered in this paper as having an impact on the employee's ability to take advantage of an Empowerment Enabling Environment are high Core self evaluation, high Risk taking, high Pro-activeness, high Self-efficacy and an internal Locus of Control. Such a personality has been referred to as an 'Empowerment Enabling Personality' in this paper.

What companies look for in terms of outcomes of an empowered work culture in employees are higher levels of effectiveness at work, their levels of innovation, leadership skills, commitment to the organization and their ability to manage stressful situations at the workplace. We shall refer to them as 'Desirable organizational outcomes'.

The model proposed in this paper can be simplistically represented as shown in Figure 1. An Empowerment Enabling Environment leads to Desirable Organizational Outcomes, but this relationship is moderated by the factor of individual personalities. Thus, an Empowerment Enabling Personality is found to facilitate the Desirable Outcomes and acts as a moderating variable. It must be noted that the terms Empowerment Enabling Environment, Empowerment Enabling Personality and Desirable Organizational Outcomes are used strictly in the context of their definitions in this paper and are not indicative of anything else. The elements that define these terms have been derived based on literature review as described in the following section. 


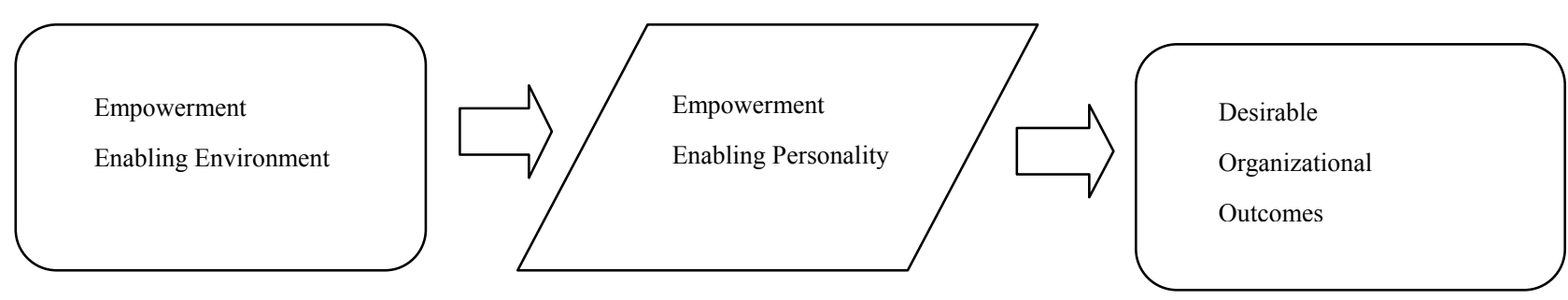

Figure 1. Representative model for organizational empowerment

\section{Literature Review}

\subsection{Defining Empowerment}

Empowerment has been described as a means to enable employees to make decisions (Bowen \& Lawler, 1992) and as a personal phenomenon where individuals take responsibility for their own actions (Pastor, 1996). According to Quinn and Spreitzer (1999), empowered people have a sense of self-determination, meaning, competence and impact.

According to Pardo del Val and Lloyd (2003), Empowerment is the management style where managers share with the rest of the organizational members their influence in the decision making process - that is to say, the collaboration in the decision making process is not limited to those positions with formal power -, with certain characteristics as far as information systems, training, rewarding, power sharing, leadership style and organizational culture concerns.

Lashley (1996) defines empowerment for using the strategy, this is in relation to an organization's purpose. Organizations may focus on a specific empowerment aim at the price of other potential gains from seeking other empowerment goals. Pursuit of one objective does not automatically exclude the others.

\subsection{The Need for Empowerment}

Employees at organizations face uncertainty, change, complexity and a high amount of work pressure. Some of the factors causing this are: demand for higher quality and value for money, a better work life balance, efforts to contain growth, equal opportunities for all and ecological issues and awareness of inequities in the global economic system; and in the past few years - international recession. Organizations need to find ways, so that they could succeed - first, by releasing creative energy, intelligence and initiative and second, by learning how to bond employees in solving workplace issues, achieving common goals, equipped with a sense of accomplishment and valuing differences of each and every employee. Organizations, which follow this, will have a stronger chance of succeeding and prospering in the global market. There are stronger chances of attracting highly competent employees and have stronger relationships with clients and customers. (Block, 1993; Harman \& Harman, 1990).

Quinn and Spreitzer's (1999) study also supports the above, they also found that empowered employees see themselves as more effective and efficient in their work. They also see themselves to try out new things or create more innovative ways of doing things. They are also transformational in their leadership abilities.

A research by Seibert, Wang and Courtright (2011) provides strong support for why managers should match claims with reality when it comes to empowerment. Research done on 142 prior studies, reveals the most effective means to achieve employee empowerment for an organization is at its workplace, itself. One of the studies revealed that for both employees and teams, empowerment drives both employee performances and attitudes. Empowered employees were more satisfied and committed at work and less likely to experience stress and anxiety and do not think about quitting the work scenario. Some of the critical behaviors driven by empowerment were performance, innovation, and organizational citizenship. A growth in revenue and profit is what empowered employees produce in a healthy work environment.

\subsection{Achieving Empowerment}

Margaret Erstard (1997) approaches the issue of employee empowerment from various angles. According to her, Empowerment is not something, which can be tossed over from management or supervisor to employee. It is relatively a complex procedure, which necessitates a clear vision, an a invigorating learning atmosphere for both management, supervisors and employees. Nevertheless, participation and implementation tools and techniques must be in place in order to be successful. 
Empowerment strategy described by Nixon (1994) - a five point one, in order to develop an organization where employees can work individually and in teams towards attainment of common goals. The strategy consists of:

- $\quad$ establishing a clear cut;

- prioritizing and acting depending on the level of the impact;

- forming strong relationships with coworkers and colleagues;

- $\quad$ expanding networks;

- $\quad$ using both internal and external support teams.

But how does one ultimately measure whether employee empowerment has been achieved or not? Quinn and Spreitzer (1999) suggest that the following characteristics reflect the personal experience of the feeling of empowerment:

- Empowered people have a sense of self-determination;

- Empowered people have a sense of meaning;

- Empowered people have a sense of competence;

- Empowered people have a sense of impact.

Few researchers have defined personality as 'an individuals' characteristic patterns of thought, emotion, and behaviour, together with the psychological mechanisms -- hidden or not -- behind those patterns'. Personality is a pattern of relatively permanent traits and unique characteristics that give both consistency and individuality to a person's behaviour." (Feist \& Feist, 2008).

Relatively little research however has gone into understanding the impact of personality on organization's efforts to empower its employees. In a 2011 study by Deborah Kaylee Ford which included 252 nurses from union organizations in Oregon, Florida, and Missouri it was found that task performance, perceived effectiveness, and satisfaction with quality of care improved when nurses were high on either proactive personality or when a nurse was low on both variables in the model did they show reduced benefits.

According to Seibert, S. E., Wang, G, and Courtright, S. H. (2011), an employee personality like core self-evaluation, self-efficacy, locus of control is intensely related with either structural or psychological empowerment. Those high on either proactive personality or political skill had higher levels of task performance and satisfaction. Even those high on either structural empowerment or political skill alos had higher levels of task performance and satisfaction. A practical consequence of the outcome is that organizations could probably consider selecting employees who have affirmative self-evaluation traits to help create a workforce that is more willing and competent and take an active role in improving one's own performance.

\section{Methodology}

A comprehensive questionnaire was developed based on the literature review and hypothesized model. Random sampling was used. The questionnaire consisted of three parts:

1) Questions on personality type that measured core self evaluation, self-efficacy, risk taking abilities, pro-activeness and an internal locus of control. This was based on a tool developed by Judge, Erez, Bono and Thoreson (2003).

2) Questions on Organizational Conditions that promote empowerment of employees based on Nixon's (1994) study, among others mentioned in the literature review. Questions ranged from whether the employees fully understood the top management's vision and strategic direction for the organization, whether the organization gives them the opportunity to produce results that are not only favourable to the organization goals but also align with their individual needs, whether skills, knowledge and information flow freely, without being inhibited by traditional hierarchies, etc.

3) The employees' perceptions about their own effectiveness at work, their levels of innovation, leadership skills, commitment to the organization and their ability to manage stressful situations at the workplace. This was largely measured through situational questions like 'I am confident in my ability to influence my peers and subordinates', 'I am able to handle stress well at work', 'I would be very happy to spend a major part of my career in this organization', etc. The questionnaire used the interval scale format of a five-point Likert scale to record responses ranging from 'Strongly Agree' to 'Strongly Disagree'.

The questionnaire was circulated online among entry-level employees with greater than a year of work experience in the same organization across companies and sectors in India. Post-graduate students who had worked previously for more than a year were also asked to participate. In case employees had switched organizations recently, they 
were asked to answer based on how they felt about the issues during their last weeks in the previous company. A total of 243 responses were collected over a period of 3 weeks and subsequently analyzed.

\section{Findings \& Discussion of Results}

Table 1. Correlation between empowerment enabling organizational environment and employee's perceived effectiveness

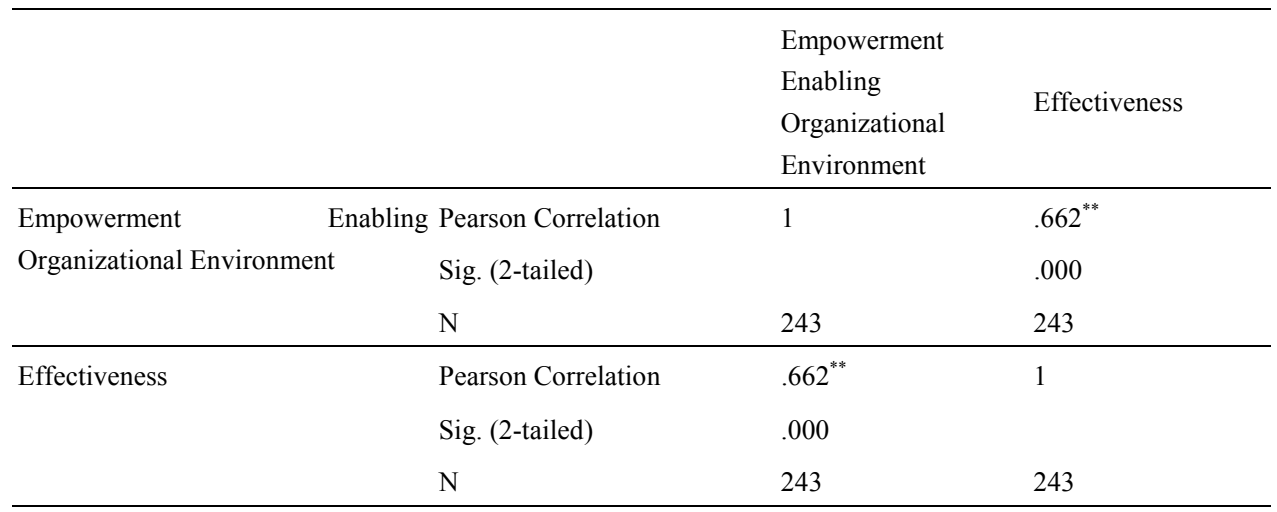

Note. $* *$ Correlation is significant at the 0.01 level (2-tailed).

Table 2. Correlation between personality-organization mismatch and employee's perceived effectiveness

\begin{tabular}{|c|c|c|c|}
\hline & & $\begin{array}{l}\text { Personality-Organizat } \\
\text { ion mismatch }\end{array}$ & Effectiveness \\
\hline \multirow[t]{3}{*}{ Personality-Organization mismatch } & Pearson Correlation & 1 & $-.609^{* *}$ \\
\hline & Sig. (2-tailed) & & .000 \\
\hline & $\mathrm{N}$ & 243 & 243 \\
\hline \multirow[t]{3}{*}{ Effectiveness } & Pearson Correlation & $-.609^{* *}$ & 1 \\
\hline & Sig. (2-tailed) & .000 & \\
\hline & $\mathrm{N}$ & 243 & 243 \\
\hline
\end{tabular}

Note. ${ }^{* *}$. Correlation is significant at the 0.01 level (2-tailed).

Table 3. Pearson correlation value (2-tailed) showing correlation coefficient between 'empowerment enabling environment' and the five desirable organizational outcomes

\begin{tabular}{ll}
\hline $\begin{array}{l}\text { Desirable } \\
\text { Outcomes }\end{array}$ & $\begin{array}{l}\text { Pearson Correlation } \\
\text { (at } \mathbf{0 . 0 1} \text { level or } \\
\text { 2-tailed) }\end{array}$ \\
\hline Effectiveness at Work & 0.662 \\
Innovation & 0.512 \\
Leadership & 0.560 \\
Commitment to organization & 0.659 \\
Stress Management & 0.236 \\
\hline
\end{tabular}


Table 4. Pearson correlation value (2-tailed) showing correlation coefficient between 'personality- organization environment mismatch' score and the five desirable organizational outcomes

\begin{tabular}{ll}
\hline Desirable Organizational Outcomes & $\begin{array}{l}\text { Pearson Correlation } \\
\text { (at 0.01 level or 2-tailed) }\end{array}$ \\
\hline Effectiveness at Work & -0.609 \\
Innovation & -0.421 \\
Leadership & -0.434 \\
Commitment to organization & -0.469 \\
Stress Management & -0.170 \\
\hline
\end{tabular}

Table 5. Pearson correlation value (2-tailed) showing correlation coefficient between the absolute value of 'personality- organization environment mismatch' score and the five desirable organizational outcomes

\begin{tabular}{ll}
\hline Desirable Organizational Outcomes & $\begin{array}{l}\text { Pearson Correlation } \\
\text { (at 0.01 level or 2-tailed) }\end{array}$ \\
\hline Effectiveness at Work & -0.156 \\
Innovation & -0.132 \\
Leadership & -0.108 \\
Commitment to organization & -0.174 \\
Stress Management & -0.165 \\
\hline
\end{tabular}

This section examines the type of responses obtained from the participants as well as the findings based on the objective of this paper.

'Empowerment Enabling Personality' is a score obtained based on the 1st part of the questionnaire, whereas 'Empowerment Enabling Environment' is a score obtained based on responses to the 2nd part of the questionnaire. The five Desirable Organizational Outcomes are taken separately for the analysis.

The Pearson's Correlation was calculated at the 0.01 level ( 2 - tailed) and it has been found that apart from Stress Management (Correlation coefficient of 0.236), all other Desirable Organizational Outcomes - Effectiveness at work (0.662), levels of Innovation (0.512), Leadership skills (0.560), Commitment to the organization (0.659) show statistically significant levels of positive correlation with 'Empowerment Enabling Organization Environment'. (Refer Table 3)

This essentially means that when the organization provides an environment to its employees which was conducive to empowerment as defined earlier and which formed the basis for the second part of the questionnaire, such as- the employees fully understood the top management's vision and strategic direction for the organization, whether the organization gives them the opportunity to produce results that are not only favourable to the organization goals but also align with their individual needs, whether skills, knowledge and information flow freely, without being inhibited by traditional hierarchies, etc.- the employees perceive themselves to be more effective at work, more innovative in their roles, have greater leadership capabilities and feel more committed to the organization. Likewise, where such empowerment enabling conditions are missing, employees perceive themselves to be less effective at work, less innovative in their roles, have fewer leadership capabilities and feel less committed to the organization. For the factor of Stress Management, the linkage was not found to be significantly strong enough to be conclusive enough and would require further research.

Coming to the aspect of personality, a 'Personality- Organization Environment Mismatch' score was developed which was essentially a difference between the 'Empowerment Enabling Personality' and the 'Empowerment Enabling Environment'. A high positive score indicates a high lack of alignment between personality type and organization environment in terms of employee's empowerment.

Once again, apart from Stress Management (Correlation coefficient of -0.170), all other Desirable Organizational Outcomes - Effectiveness at work (-0.609), levels of Innovation (-0.421), Leadership skills (-0.434), Commitment to the organization (-0.469) show statistically significant levels of negative correlation (Refer Table 4).

This part of the findings essentially checks what happens when there is a difference in the environment that the organization provides in terms of employee empowerment, and the personality of the employees in terms of how empowerment-oriented they are. The assumption is that employees who have high levels of core self evaluation, self-efficacy, risk taking abilities, pro-activeness and an internal locus of control are more empowerment-oriented. The results show that when this mismatch of organizational conditions and employee personality is high, the 
employees' perceptions about their Effectiveness at work, levels of Innovation, Leadership skills, Commitment to the organization also go down. Once again, for the factor of Stress Management, the linkage was not found to be significantly strong enough to be conclusive enough and would require further research. In other words, when organizations are not able to match empowerment-orientated employees with an empowerment oriented workplace, the levels of desirable outcomes fall sharply.

But is the reverse true as well? That is, what happens when employees who are low on 'Empowerment Enabling Personality' are provided with an empowering environment? Does effectiveness, innovation, etc fall again due to this kind of misalignment? If this were true, then the correlation of the absolute values of 'PersonalityOrganization Environment Mismatch' score with the Desirable Organizational Outcomes would be more negative as we would consider any kind of misalignment to have an equally undesirable effect. But results show otherwise. When the absolute values are correlated, the negative correlation is relatively less for all the Desirable Organizational Outcomes - Effectiveness at work (-0.156), levels of Innovation (-0.132), Leadership skills $(-0.108)$, Commitment to the organization (-0.174) and Stress Management (-0.165). (Refer Table 5)

These relatively smaller degrees of negative correlation (of the absolute values of 'Personality- Organization Environment Mismatch' score with the Desirable Organizational Outcomes compared to the higher values of negative correlation in the case of the real values of 'Personality- Organization Environment Mismatch' score with the Desirable Organizational Outcomes) essentially mean that the negative effect of a difference in personality-environment mismatch in the case of more empowerment-oriented employees is greater than in the case of less empowerment-oriented employees.

These results clearly have significant implications on how organizations manage their Human Resource policies and practices.

\section{Implications}

The findings above provide some clear indications. Firstly, it makes business sense to go for an empowered work environment. The research clearly indicates that organizations, which provide employees with conditions that enable empowerment are rewarded with employees who are significantly more committed, show greater leadership potential, and see themselves as more effective and innovative. Though the implications on stress management are not so high, a slight positive correlation still exists and this aspect may be explored further in subsequent research.

More importantly, the study has major implications on the human capital strategy of organizations. Often hiring managers are told to go after candidates who show great potential in terms of their personality to make it big in the corporate world. And so, we have organizations going all out to attract people displaying high levels of core self evaluation, self-efficacy, risk taking abilities, pro-activeness and an internal locus of control. But what happens when such high potential candidates actually join the organization and find an environment that is not conducive to their ambitious and self-confident personality? When they are forced to do jobs which they have little personal inclination towards, have no autonomy when it comes to making everyday decisions at work, have no sense of direction about where the company is headed and what is their role in it- they end up performing way below their potential. While they may soon leave the organization because they don't want to jeopardize their own careerwhat happens to the organization which refuses to appreciate that even the best of employees cannot deliver in an environment that is stifling and restricting?

At the same time, what is also interesting is the finding that even those employees who do not show a great propensity towards empowerment based purely on their personality profile do not underperform in an empowering environment. Clearly, an organization that enables and sustains an empowered culture stands to benefit from more satisfied employees and higher performance. The practical implication of course is that organizations need to recruit and select employees high on core self evaluation, etc and provide them with an empowering culture in order to achieve best results.

In today's competitive talent market, where organizations are increasingly looking for innovative, self-motivated employees in a flatter organizational context that defies traditional norms of hierarchy and chronological seniority, the provision or lack of empowerment can have serious impact on its long term performance and growth, besides its reputation as an employer of choice. The findings about personality's influence will also be seen as increasingly significant as more individuals of Generation $\mathrm{Y}$ and $\mathrm{Z}$ enter the workforce and their aspirations and personal objectives can be met by an empowered workplace, which can help unleash their creative potential. On the other hand, a lack of the same can lead to low engagement and high turnover. 


\section{Conclusions}

The importance of employee empowerment in the organization has been clearly established through literature review and a survey of 243 employees which has shown that organizational empowerment conditions - which can be achieved by ensuring that employees are in alignment with the organization's mission and vision, they are given the opportunity to produce results that have a personal appeal to them, where traditional hierarchies do not obstruct the free flow of information both laterally and vertically, where people share mutually satisfying relationships, they are given the freedom to make quick decisions related to their work, and are given opportunities to participate, network and voice their opinions and concerns- lead to higher levels of effectiveness at work, greater innovation, leadership skills, commitment to the organization. A small, but statistically insignificant positive correlation has been shown with stress management as well.

The research also moved into the realm of individual personality and the impact this factor can have on organization's attempts to empower their employees in order to achieve better results. In this regard, the study has shown us that employees with higher levels of core self evaluation, self-efficacy, risk taking abilities, pro-activeness and an internal locus of control require more empowered spaces so that their potential can meet the actual performance. When the levels of empowerment for such employees are low, they score low on parameters such as effectiveness, innovation, leadership skills, commitment and stress management. Additionally, employees with relatively low levels of core self evaluation, self-efficacy, risk taking abilities, pro-activeness and an external locus of control do not fare badly in an empowered environment. These findings can thus be used to conclude that a more empowered work environment benefits both employers and all kinds of employees.

\section{References}

Block, P. (1993). Stewardship-Choosing Service over Self-interest. Benett-Koehler, San Francisco, CA.

Bowen, D. E., \& Lawler, E. E. (1992). The empowerment of service workers: what, why, how and when. Sloan Management Review, 33(3).

Del Val, M. P., \& Lloyd, B. (2003). Measuring empowerment. Leadership \& organization development journal, 24(2), 102-108.

Feist, J., \& Gregory, J. F. (2002). Theories of Personality (5th ed.). Boston: McGraw-Hill.

Ford, D. K. (2011). An Evaluation of Moderating Influences of Employee Proactive Personality: Empowerment and Political Skill. Portland State University.

Harman, W., \& Harmann, J. (1990). Creative Work, Knowledge Systems Inc.

Judge, T. A., Erez, A., Bono, J. E., \& Thoresen, C. J. (2003). The core self-evaluations scale: Development of a measure. Personnel Psychology, 56(2), 303-331.

Lashley, C. (1996). Research issues for employee empowerment in hospitality operations. International Journal of Hospitality Management, 15(3), 283-298.

Margaret, E. (1997). Empowerment and organizational change. International Journal of Contemporary Hospitality Management.

Nixon, B. (1994). Developing an empowering culture in organizations. Empowerment in Organizations, 2(1). http://dx.doi.org/10.1108/09684899410054634

Pastor, J. (1996). Empowerment: what it is and what it is not. Empowerment in Organizations, 4(2). http://dx.doi.org/10.1108/09684899610118028

Quinn, R. E., \& Spreitzer, G. (1999). The road to empowerment: seven questions every leader should consider. IEEE Engineering Management Review, 27, 21-28. http://dx.doi.org/10.1016/S0090-2616(97)90004-8

Seibert, S. E., Wang, G., \& Courtright, S. H. (2011). Antecedents and consequences of psychological and team empowerment: A meta-analytic review. Journal of Applied Psychology. http://dx.doi.org/10.1037/a0022676

\section{Copyrights}

Copyright for this article is retained by the author(s), with first publication rights granted to the journal.

This is an open-access article distributed under the terms and conditions of the Creative Commons Attribution license (http://creativecommons.org/licenses/by/3.0/). 